\title{
ARTICLE OPEN Nodeless superconductivity and its evolution with pressure in the layered dirac semimetal $2 \mathrm{M}-\mathrm{WS}_{2}$
}

Zurab Guguchia', Dariusz J. Gawryluk², Marta Brzezinska ${ }^{3,4}$, Stepan S. Tsirkin ${ }^{3}$, Rustem Khasanov ${ }^{1}$, Ekaterina Pomjakushina ${ }^{2}$, Fabian O. von Rohr ${ }^{3,5}$, Joel A. T. Verezhak ${ }^{1}$, M. Zahid Hasan $\left(\mathbb{D}^{6}\right.$, Titus Neupert ${ }^{3}{ }^{3}$, Hubertus Luetkens ${ }^{1}$ and Alex Amato ${ }^{1}$

Recently, the transition metal dichalcogenide (TMD) system $2 \mathrm{M}-\mathrm{WS}_{2}$ has been identified as a Dirac semimetal exhibiting both superconductivity with the highest $T_{\mathrm{c}} \sim 8.5 \mathrm{~K}$ among all the TMD materials and topological surface states. Here we report on muon spin rotation $(\mu \mathrm{SR})$ and density functional theory studies of microscopic SC properties and the electronic structure in $2 \mathrm{M}-\mathrm{WS}_{2}$ at ambient and under hydrostatic pressures $\left(p_{\max }=1.9 \mathrm{GPa}\right)$. The $\mathrm{SC}$ order parameter in $2 \mathrm{M}-\mathrm{WS}_{2}$ is determined to have single-gap $s$ wave symmetry. We further show a strong negative pressure effect on $T_{c}$ and on the SC gap $\Delta$. This may be partly caused by the pressure induced reduction of the size of the electron pocket around the $\Gamma$-point. We also find that the superfluid density $n_{s}$ is weakly affected by pressure. The absence of a strong pressure effect on $n_{s}$ and the absence of a correlation between $n_{s}$ and $T_{c}$ in $2 \mathrm{M}-\mathrm{WS}_{2}$, in contrast to the other SC TMDs $T_{\mathrm{d}}-\mathrm{MoTe}_{2}$ and $2 \mathrm{H}-\mathrm{NbSe}_{2}$, is explained in terms of its location in the optimal (ambient pressure) and above the optimal (under pressure) superconducting regions of the phase diagram and its large distance to the other possible competing or cooperating orders.

npj Quantum Materials (2019)4:50 ; https://doi.org/10.1038/s41535-019-0189-5

\section{INTRODUCTION}

The transition metal dichalcogenides (TMDs) are representative layered materials presently attracting very strong interest from research communities working on nanoscience and 2-d layered systems. ${ }^{1-14}$ The TMDs share the $\mathrm{MX}_{2}$ chemical formula, with $\mathrm{M}$ being a transition metal ( $\mathrm{M}=\mathrm{Ti}, \mathrm{Zr}, \mathrm{Hf}, \mathrm{V}, \mathrm{Nb}, \mathrm{Ta}, \mathrm{Mo}, \mathrm{W}$ or $\mathrm{Re}$ ) and $X$ being a chalcogen $(X=S, S e$, or Te). TMDs are obtained in various crystal structures $1 \mathrm{~T}, 2 \mathrm{H}$, and $1 \mathrm{~T}^{\prime}$ with different stackings of the individual $\mathrm{MX}_{2}$ layers. A number of ground states have been observed in these systems including charge-density waves, superconductivity, semiconductors and topological metals. New physics findings in TMDs include strong excitonic states, novel phenomena from valley splitting, Dirac cone dispersion and topological effects, and more. ${ }^{1,4}$

Layered materials, with highly anisotropic electronic properties have been found to be potential hosts for unconventional superconductivity. TMDs exhibit many features strikingly similar to those reported in other unconventional superconductors. Superconductivity can be observed under pressure as for the pristine 1T polytype TMD materials which are not superconducting at ambient pressure, but exhibit charge density wave (CDW) states, instead. ${ }^{15}$ The metallic $2 \mathrm{H}$ polytypes have two, or more, Fermi surfaces and saddle bands, allowing for dual orderings, which can be coexisting CDW and SC orderings, two superconducting gaps, two CDW gaps, and possibly even pseudogaps above the onset temperature of CDW orderings, as reported in some cuprate superconductors. ${ }^{16-18}$ Upon application of pressure, the CDW ground state is suppressed and the superconducting transition temperature is enhanced. A dome-like superconducting phase was induced by electrostatic gating in the semiconducting $2 \mathrm{H}-\mathrm{MoSe}_{2}, 2 \mathrm{H}-\mathrm{MoS}_{2}, 2 \mathrm{H}-\mathrm{MoTe}_{2}$, and $2 \mathrm{H}-\mathrm{WS}_{2}$ with a $T_{\mathrm{c}}$ of up to $10 \mathrm{~K}^{19-21}$ It was also shown that using field effect gating, one can induce superconductivity in monolayer semiconducting TMD $\mathrm{WS}_{2}{ }^{22}$ and one could access an unprecedented doping range revealing a rich set of competing electronic phases ranging from band insulator, via superconductor, to a reentrant insulator at high doping. Superconductivity with a very low $T_{\mathrm{c}} \sim 0.1 \mathrm{~K}$ was also reported in the Weyl semimetal $\mathrm{MoTe}_{2}$ which exhibits a low temperature orthorhombic $T_{\mathrm{d}}$ structure. ${ }^{5}$ However, under the application of pressure, the orthorhombic structure transforms into the $1 \mathrm{~T}^{\prime}$ structure and $T_{\mathrm{c}}$ is enhanced to about $8 \mathrm{~K}$. Recently, we found a correlation between the superfluid density at $T=0$ and the transition temperature $T_{\mathrm{c}}$ in the type-II Weyl semimetal $T_{d^{-}}$ $\mathrm{MoTe}_{2}{ }^{23}$ and the deviation from the linear correlation in $2 \mathrm{H}-\mathrm{NbSe}_{2}$ under pressure. ${ }^{24}$ These observations resemble the ones found earlier in cuprates ${ }^{25-30}$ and Fe-based superconductors. ${ }^{31-34}$ These examples demonstrate that the TMDs exhibit rather unconventional superconducting and normal state properties.

Recently, superconductivity with $T_{\mathrm{C}} \sim 8.8 \mathrm{~K}$ was reported in the new compound $2 \mathrm{M}-\mathrm{WS}_{2}$, which is constructed from $1 \mathrm{~T}^{\prime}-\mathrm{WS}_{2}$ monolayers. ${ }^{35} 2 \mathrm{M}-\mathrm{WS}_{2}$ has the highest $T_{\mathrm{c}}$ among all TMD materials at ambient pressure. The system possesses a semimetallic band structure. The crystal structure of $2 \mathrm{M}-\mathrm{WS}_{2}$ has a different stacking of $1 \mathrm{~T}^{\prime}-\mathrm{WS}_{2}$ monolayers along the a-axis, compared to $T_{\mathrm{d}}-\mathrm{MoTe}_{2}$ and $1 \mathrm{~T}^{\prime}-\mathrm{WTe}_{2}$. In addition to superconductivity, topological surface states with a single Dirac cone were theoretically predicted for $2 \mathrm{M}-\mathrm{WS}_{2}$. Hence, this system represents one of the few examples of a material with both superconductivity and a topologically non-

\footnotetext{
${ }^{1}$ Laboratory for Muon Spin Spectroscopy, Paul Scherrer Institute, CH-5232 Villigen PSI, Switzerland; ${ }^{2}$ Laboratory for Multiscale Materials Experiments, Paul Scherrer Institut, 5232

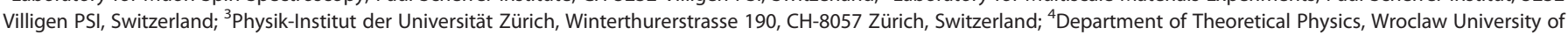

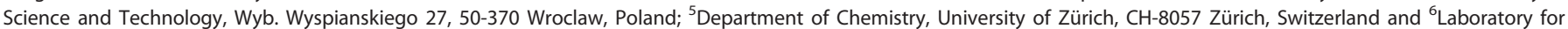
Topological Quantum Matter and Spectroscopy, Department of Physics, Princeton University, Princeton, NJ 08544, USA Correspondence: Zurab Guguchia (zurab.guguchia@psi.ch) or Dariusz J. Gawryluk (dariusz.gawryluk@psi.ch)
}

Received: 4 April 2019 Accepted: 13 August 2019

Published online: 10 September 2019 

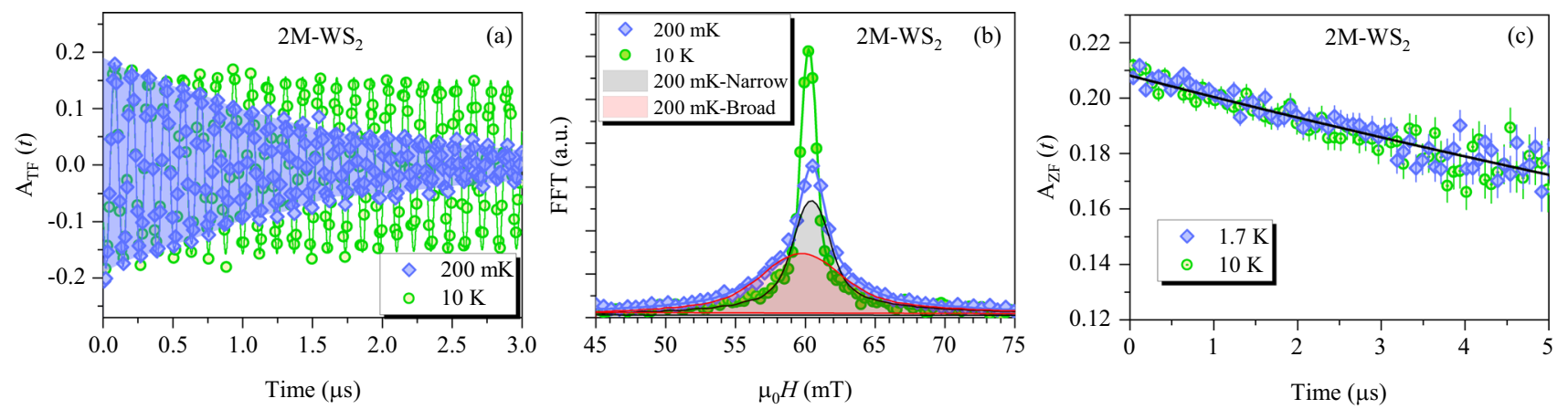

Fig. 1 Transverse-field (TF) and Zero-field (ZF) $\mu$ SR time spectra for $2 \mathrm{M}-\mathrm{WS}_{2}$. a The TF spectra, above and below $T_{c}$ are shown. The solid lines represent fits to the data by means of Eq. (1); b The Fourier transforms of the $\mu$ SR time spectra shown in a. c ZF $\mu$ SR time spectra for $2 \mathrm{M}-\mathrm{WS} \mathrm{S}_{2}$ recorded above and below $T_{c}$. The line represents the fit to the data of the combination of Lorentzian and Gaussian Kubo-Toyabe depolarization function reflecting the field distribution at the muon site created by nuclear dipole moments and randomly oriented diluted weak local electronic moments. Error bars are the s.e.m. in about $10^{6}$ events. The error of each bin count $n$ is given by the s.d. of $n$. The errors of each bin in $A(t)$ are then calculated by s.e. propagation

trivial surface states. The possible occurrence of topological superconductivity (TSC) in $2 \mathrm{M}^{-W_{2}}$ after fine tuning of some parameters is also discussed. ${ }^{35}$ TSCs are materials with unique electronic states consisting of a full pairing gap in the bulk and gapless surface states composed of Majorana fermions (MFs). ${ }^{36-38}$ Up to now, the only known properties of the superconducting state in $2 \mathrm{M}-\mathrm{WS}_{2}$ are the critical temperatures and fields. ${ }^{35}$ Thus, a thorough exploration of superconductivity in $2 \mathrm{M}-\mathrm{WS}_{2}$ from both experimental and theoretical perspectives is imperative.

To further study superconductivity and its nature in $2 \mathrm{M}-\mathrm{WS}_{2}$, it is critical to measure the superconducting order parameter at the microscopic level. Thus, we conducted ambient and highpressure ${ }^{39-42}$ muon spin relaxation/rotation $(\mu S R)$ measurements of the magnetic penetration depth $\lambda$ in the polycrystalline sample of $2 \mathrm{M}-\mathrm{WS}_{2}$. This quantity is one of the fundamental parameters of a superconductor, since it is related to the superfluid density $n_{s}$ via $1 / \lambda^{2}=\mu_{0} e^{2} n_{s} / m^{*}$ [where $m^{*}$ is the effective mass; see also Eq. (2)]. Most importantly, the temperature dependence of $\lambda$ is particularly sensitive to the topology of the superconducting gap: while in a fully gapped superconductor, $\Delta \lambda^{-2}(T) \equiv \lambda^{-2}(0)-\lambda^{-2}(T)$ vanishes exponentially upon decreasing the temperature, it vanishes as a power of $T$ in a superconductor containing a gap with nodes. The $\mu \mathrm{SR}$ technique provides a powerful tool to measure $\lambda$ in the vortex state of type II superconductors in the bulk of the sample, ${ }^{43}$ in contrast to many techniques that probe $\lambda$ only near the surface. Details are provided in the Methods section.

Here we show that the superconducting order parameter in $2 \mathrm{M}$ $W_{2}$ possesses single-gap s-wave symmetry, which is consistent with the presence of dominant hole-type charge carriers, as deduced from Hall effect measurements. Moreover, we observed a strong negative pressure effect on the critical temperature $T_{c}$ and the superconducting gap $\Delta$ as well as on the size of the electron pocket around the $\Gamma$-point, at which a band inversion appears up to the highest applied pressure. However, the pressure effect on the superfluid density in $2 \mathrm{M}-\mathrm{WS}_{2}$ is very weak. We find that the ratio $T_{\mathrm{c}} / n_{s}$ is located in the Uemura plot close to those of other superconducting TMD systems, as $T_{\mathrm{d}}-\mathrm{MoTe}_{2}$ and $2 \mathrm{H}-\mathrm{NbSe}_{2}$, and those of other unconventional superconductors. This suggests that $2 \mathrm{M}-\mathrm{WS}_{2}$ exhibits rather unconventional superconducting properties. The absence of a strong pressure effect on the superfluid density and its correlation with $T_{\mathrm{c}}$, which was previously found in $T_{\mathrm{d}}-\mathrm{MoTe}_{2}$ and $2 \mathrm{H}-\mathrm{NbSe}_{2}$, is explained in terms of the absence of competing or cooperating order to superconductivity, as $2 \mathrm{M}-\mathrm{WS}_{2}$ exhibits optimal superconducting conditions already at ambient pressure. By application of pressure we are pushing the system away from any possible competing/cooperating order. Our findings, therefore, pose a challenge for understanding the underlying physics in these layered TMDs and might ultimately lead to a better understanding of generic aspects of non-BCS behaviors in unconventional superconductors.

\section{RESULTS}

Magnetic penetration depth and the superconducting gap symmetry

Figure 1a exhibits transverse-field (TF) muon-time spectra for the polycrystalline sample of $2 \mathrm{M}-\mathrm{WS}_{2}$ measured in an applied magnetic field of $\mu_{0} \mathrm{H}=60 \mathrm{mT}$ above $(T=10 \mathrm{~K})$ and below $(T=$ $200 \mathrm{mK}$ ) the superconducting (SC) transition temperature $T_{c}$. Above $T_{c}$ the oscillations reflecting the muon-spin precession show a small damping due to the random local fields from the nuclear magnetic moments. Below $T_{\mathrm{c}}$ the relaxation rate strongly increases upon decreasing the temperature due to the presence of a nonuniform local magnetic field distribution resulting from the formation of the flux-line lattice (FLL) in the Shubnikov phase. By carefully examining the TF- $\mu$ SR data, we find that the sample consists of a superconducting and a non-superconducting volume fraction. To visualise these two fractions of the sample, we report on Fig. $1 \mathrm{~b}$ the Fourier transforms of the $\mu \mathrm{SR}$ time spectra, shown in Fig. 1a. At $T=200 \mathrm{mK}$ the narrow signal around $\mu_{0} H_{\text {ext }}=60 \mathrm{mT}$, originates from the non-superconducting part of the sample, while the broad signal with a first moment $\mu_{0} H_{\text {int }}<\mu_{0} H_{\text {ext, }}$ marked by the solid arrow in Fig. 1b, arises from the superconducting part of the sample. The estimated superconducting volume fraction from the $\mu \mathrm{SR}$ data is $\sim 45 \%$. It is interesting to investigate whether magnetism is present either in the superconducting or nonsuperconducting fractions of the sample. In order to search for magnetism, we have carried out zero-field (ZF) $\mu \mathrm{SR}$ experiments above and below $T_{C}$, which are shown in Fig. 1c. No sign of static magnetism could be detected in the ZF time spectra down to $1.7 \mathrm{~K}$. Moreover, the ZF relaxation rate is small and does not change between 10 and $1.7 \mathrm{~K}$. ZF- $\mu \mathrm{SR}$ spectra are well described by the combination of a Lorentzian and a Gaussian Kubo-Toyabe depolarization function, ${ }^{44,45}$ reflecting the field distribution at the muon site created by the nuclear dipole moments and randomly oriented diluted weak local electronic moments. Returning to the discussion of the TF- $\mu$ SR data, we observe a strong diamagnetic shift of the internal magnetic field $\mu_{0} H_{\text {int }}$ sensed by the muons below $T_{c}$. This is evident in Fig. $2 \mathrm{a}$, where we plot the temperature dependence of $\Delta B_{\text {dia }}=\mu_{0}\left(H_{\text {int,SC }}-H_{\text {int,Ns }}\right)$, i.e., the difference between the internal field $\mu_{0} H_{\text {int,SC }}$ measured in the SC fraction and one $\mu_{0} H_{\text {int,Ns }}$ measured in the normal state at $T=10 \mathrm{~K}$. The strong diamagnetic shift along with the negative field dependence of the superconducting depolarization rate $\sigma_{\mathrm{sc}}$ (see Fig. 2b) excludes the occurrence of field induced magnetism in $2 \mathrm{M}-\mathrm{WS}_{2}$. Note that in some Fe-based and cuprate high temperature 

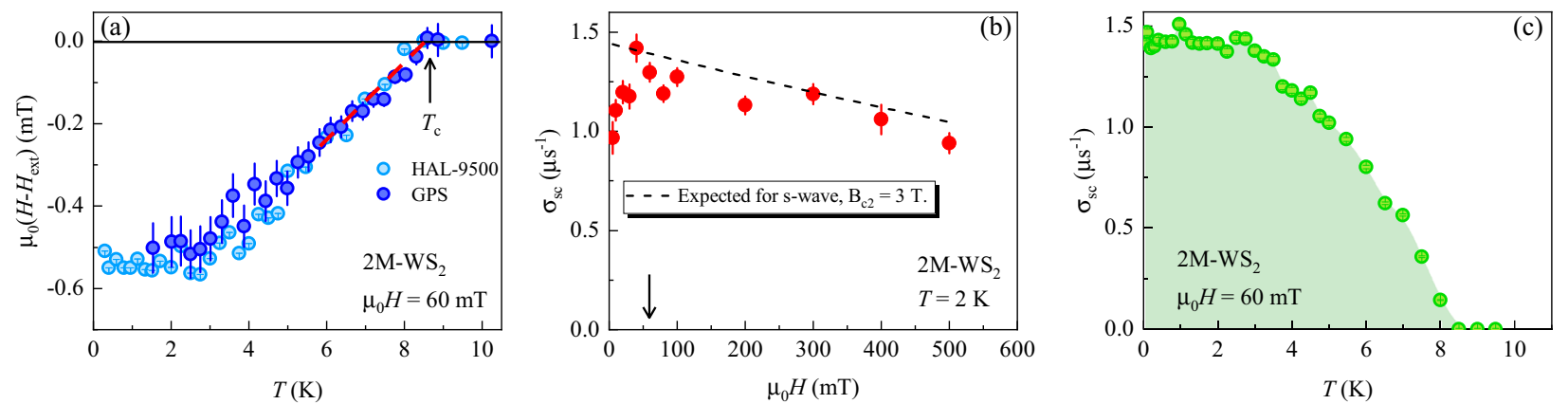

Fig. 2 Superconducting muon spin depolarization rate $\sigma_{\mathrm{sc}}$ and the diamagnetic shift for $2 \mathrm{M}-\mathrm{WS}$. a The temperature dependence of the diamagnetic shift $\Delta B_{\text {dia }}=\mu_{0}\left(H_{\text {int,SC }}-H_{\text {int, NS }}\right)$ in an applied magnetic field of $\mu_{0} H=60 \mathrm{mT}$. The arrow denotes $T_{\mathrm{c}}$. The data taken on HAL-9500 $\mu S R$ spectrometer, equipped with BlueFors vacuum-loaded cryogen-free dilution refrigerator (DR) and on the standard GPS spectrometer are shown. b Field dependence of the the muon spin relaxation rate $\sigma_{\mathrm{sc}}(T)$ at $1.6 \mathrm{~K}$. Arrow indicates the field value at which the temperature dependent measurements were carried out. Dashed line shows the theoretical field dependence expected for the $s$-wave superconductor, taking into account the critical field of $3 \mathrm{~T}$. c The temperature dependence of $\sigma_{\mathrm{sc}}(T)$. The error bars represent the s.d. of the fit parameters

superconductors, where field induced magnetism was detected, a paramagnetic shift and a linear increase of $\sigma_{\mathrm{sc}}$ with magnetic field were observed. ${ }^{46-48}$ The absence of magnetism in zero-field or under applied magnetic fields implies that the increase of the TF relaxation rate below $T_{c}$ is solely arising from the FLL in the superconducting fraction. The strong superconducting response from nearly $50 \%$ of the sample points to the bulk superconductivity in $2 \mathrm{M}-\mathrm{WS}_{2}$. We note that the diamagnetic moment, which we deduce from the bulk magnetization measurements suggest a $100 \%$ SC volume in the sample, which is consistent with the previous report. ${ }^{35}$ However, bulk magnetisation measures the diamagnetic screening of the whole sample where a $100 \%$ screening does not necessarily imply a $100 \%$ SC volume. $\mu \mathrm{SR}$, being a local probe, directly measures the SC volume fraction within the sample. Thus, in this work we provide a more precise estimate for the SC volume in $2 \mathrm{M}-\mathrm{WS}_{2}$. The $\mu \mathrm{SR}$ evidence for a phase separation between SC and paramagnetic regions in $2 \mathrm{M}$ $\mathrm{WS}_{2}$ is an interesting finding, which might be an intrinsic feature of this system.

From the TF muon time spectra, we determined the Gaussian superconducting relaxation rate $\sigma_{\mathrm{sc}}$ (after subtracting the nuclear contribution), which is proportional to the second moment of the field distribution (see Methods section). Figure $2 \mathrm{~b}$ shows the obtained field dependence of $\sigma_{\mathrm{sc}}$ at $1.7 \mathrm{~K}$. Each point was obtained by field cooling the sample from above $T_{c}$ to $1.7 \mathrm{~K}$. As expected from the London model one observes that first $\sigma_{\mathrm{sc}}$ strongly increases with increasing magnetic field until reaching a maximum at $40 \mathrm{mT}^{49}$ Above $40 \mathrm{mT}, \sigma_{\mathrm{sc}}$ decreases with increasing magnetic field. This appears consistent with a behaviour expected for $s$-wave superconductor for an ideal triangular vortex lattice. ${ }^{49}$ Taking into account the critical field of $3 \mathrm{~T}$, the theoretical s-wave behaviour has been calculated according to the Brandt formula. ${ }^{49}$ It is shown by the dashed line in Fig. 2b, which agrees well with the experimental data. The second moment of the resulting inhomogeneous field distribution is related to the magnetic penetration depth $\lambda$ by $\left\langle\Delta B^{2}\right\rangle \propto \sigma_{s c}^{2} \propto \lambda^{-4} \cdot{ }^{49}$ In order to investigate the symmetry of the superconducting gap, we have therefore derived the temperature-dependent London magnetic penetration depth $\lambda$ $(T)$, which is related to the relaxation rate by:

$$
\frac{\sigma_{\mathrm{sc}}(T)}{\gamma_{\mu}}=0.06091 \frac{\Phi_{0}}{\lambda^{2}(T)} \text {. }
$$

Here, $\gamma_{\mu}$ is the gyromagnetic ratio of the muon, and $\Phi_{0}$ is the magnetic-flux quantum. In order to accurately determine the temperature dependence of $\lambda$, it is important to do the temperature dependent measurements of $\sigma_{\mathrm{sc}}$ slightly above the maximum of $\sigma_{\mathrm{sc}}(H)$, where Eq. (1) is valid. Thus, the measurements were done

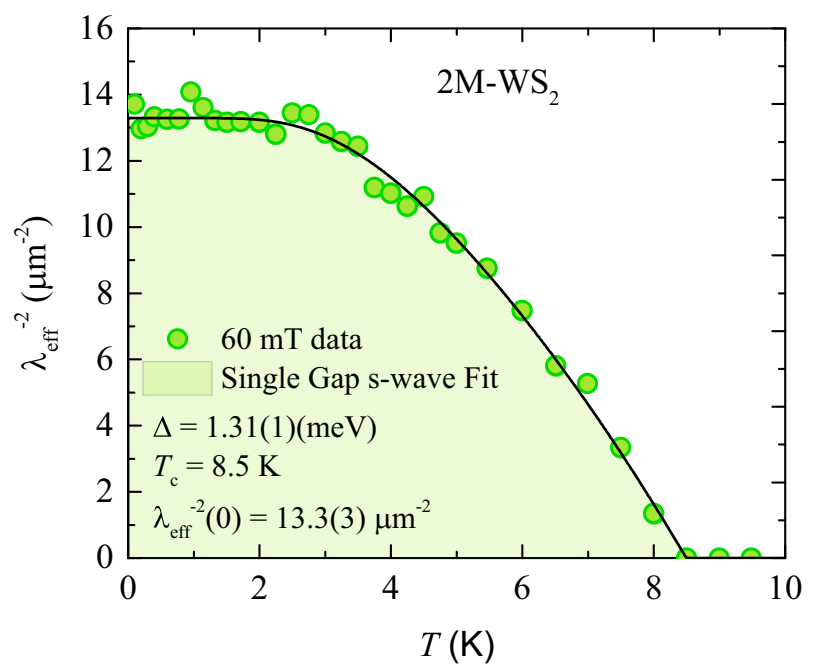

Fig. 3 Temperature evolution of $\lambda^{-2}$ for $2 \mathrm{M}-\mathrm{WS}_{2}$. a The temperature dependence of $\lambda^{-2}$ measured at ambient pressure. The solid line represents a fit using a single gap s-wave model. The error bars are calculated as the s.e.m

with an applied magnetic field of $60 \mathrm{mT}$. In Fig. 2c, we show the temperature dependence of the muon spin depolarization rate $\sigma_{\mathrm{sc}}$. Below $T_{\mathrm{c}}$ the relaxation rate $\sigma_{\mathrm{sc}}$ starts to increase from zero with decreasing temperature due to the formation of the FLL and reaches $\sigma_{\mathrm{sc}} \simeq 1.43 \mu \mathrm{s}^{-1}$ at the base- $T$. The temperature dependence of $\sigma_{\mathrm{sC}}$ which reflects the topology of the SC gap, exhibits a saturation at low temperatures. This is consistent with a nodeless superconductor, for which the superfluid density $n_{s} \propto \sigma_{s c}$ reaches its zerotemperature value exponentially. For a quantitative analysis, we in the local (London) approximation $(\lambda \gg \xi$, where $\xi$ is the coherence length), calculated $\lambda(T)$. The result of the gap analysis is shown in Fig. 3 . The data are perfectly compatible with a single $s$-wave gap with a value of $\Delta=1.31$ (1) meV.

The London magnetic penetration depth $\lambda$ is given as a function of $n_{s}, 50$ the effective mass $m^{*}, \xi$ and the mean free path $l$, according to

$\frac{1}{\lambda^{2}}=\frac{4 \pi n_{s} e^{2}}{m^{*} c^{2}} \cdot \frac{1}{1+\xi / l}$.

For systems close to the clean limit, $\xi / l \rightarrow 0$, the second factor essentially becomes unity, and the simple relation $1 / \lambda^{2} \propto n_{s} / m^{*}$ holds. Considering the upper critical fields $H_{c_{2}}$ of $2 \mathrm{M}-\mathrm{WS}_{2}$, as reported in detail by Fang et al., ${ }^{35}$ we can estimate the in-plane 

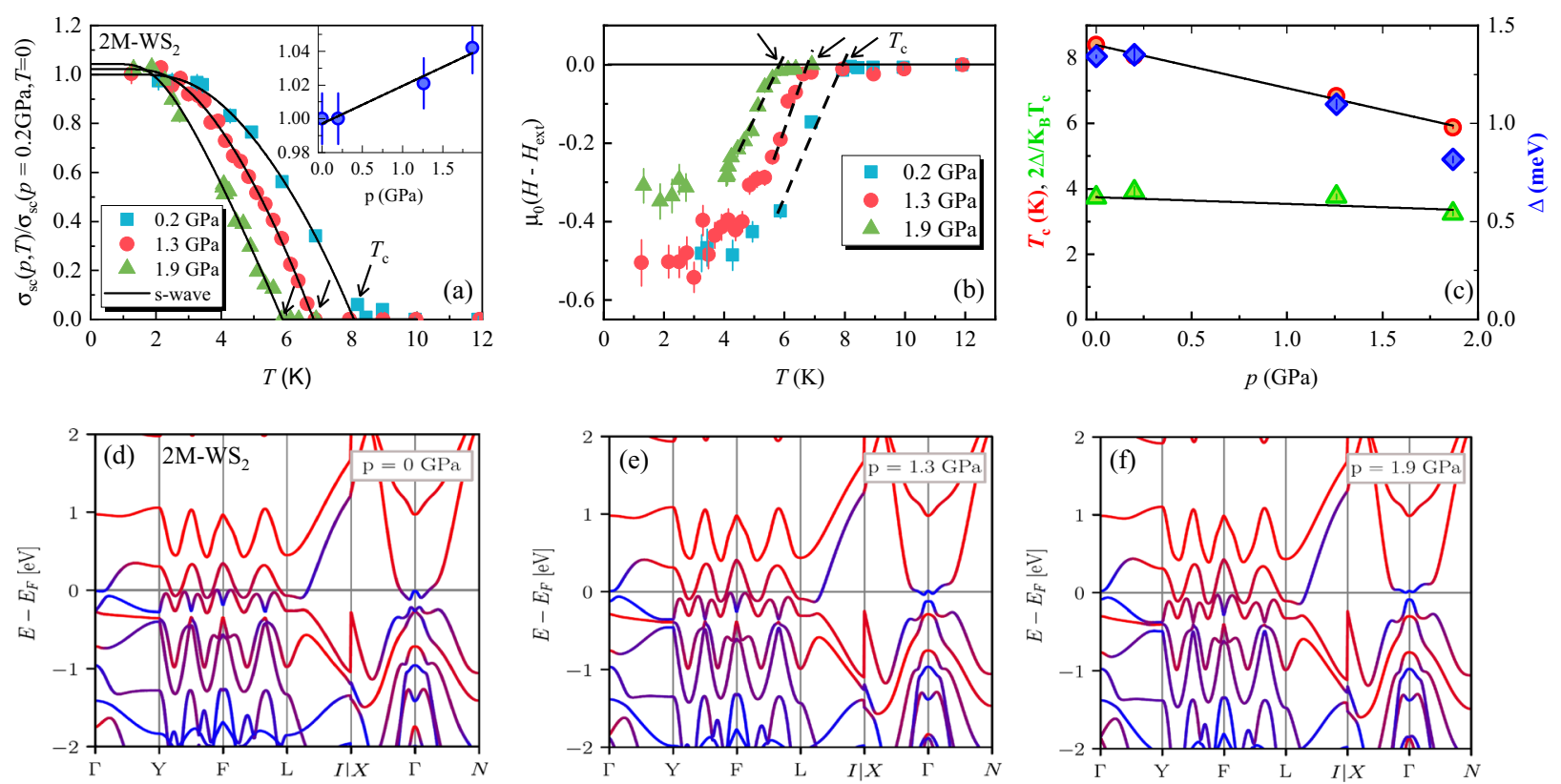

Fig. 4 Pressure evolution of various quantities. a The temperature dependence of $\sigma_{\mathrm{sc}}(T)$, normalized to its value for the lowest applied pressure, under various applied pressures. The inset illustrates the weak positive pressure dependence of $\sigma_{\mathrm{sc}}$. $\mathbf{b}$ The temperature dependence of the diamagnetic shift $\Delta B_{\text {dia }}=\mu_{0}\left(H_{\text {int,sC }}-H_{\text {int,Ns }}\right)$, recorded for various hydrostatic pressures. c Pressure dependence of the superconducting transition temperature $T_{c}$, the zero-temperature value of the superconducting gap $\Delta$ and the ratio $2 \Delta / K_{B} T_{c}$ for $2 \mathrm{M}$-WS $\mathrm{S}_{2}$. The error bars represent the s.d. of the fit parameters. Band structure in the presence of spin-orbit coupling for $p=0 \mathrm{GPa} \mathbf{d}, 1.3 \mathrm{GPa}$ e and $1.9 \mathrm{GPa}$ f. Red color corresponds to $d$ orbitals of W atoms, while the blue color indicates the $p$ orbitals of $\mathrm{S}$ atoms

coherence length to be $\xi_{a b} \simeq 8.8 \mathrm{~nm}$ at ambient pressure. At ambient pressure, the in-plane mean free path / was estimated to be $I_{a b} \simeq 200 \mathrm{~nm}$. No estimates are currently available for $I$ under pressure. However, the in-plane $/$ is most probably independent of pressure considering the fact that the pressure mostly reduces the interlayer distance due to the unique anisotropy resulting from the stacking of layers with van der Waals type interactions between them. Thus, in view of the short coherence length and relatively large $I$, we can reliably assume that $2 \mathrm{M}-\mathrm{WS}_{2}$ lies close to the clean limit. ${ }^{23,51}$ With this assumption, we obtain the groundstate value $n_{s} /\left(m^{*} / m_{e}\right) \simeq 2.8 \times 10^{26} \mathrm{~m}^{-3}$, where $m_{e}$ is the free electron mass.

\section{Probing superconductivity and band structure as a function of pressure}

To gain further insight into the superconducting properties of $2 \mathrm{M}$ $\mathrm{WS}_{2}$, the temperature dependence of the superfluid response was measured under applied pressures up to $1.9 \mathrm{GPa}$. Moreover, the pressure effect of the electronic structure was also calculated. We note that the SC volume fraction was found to be independent of pressure. In Fig. 4a, b, we show the temperature evolution of $\sigma_{\mathrm{sc}}$ normalised to the value at ambient pressure, and the diamagnetic shift $\Delta B_{\text {dia }}$ measured under various hydrostatic pressures. A single gap s-wave model describes the $\sigma_{s c}(T)$ data very well up to the highest pressure. Remarkably, both $T_{\mathrm{c}}$ and $\Delta$ decreases substantially upon application of pressure. $T_{\mathrm{c}}$ decreases from $8.5 \mathrm{~K}$ at $p=$ $0 \mathrm{GPa}$ to $6 \mathrm{~K}$ at $1.9 \mathrm{GPa}$. $\Delta$ decreases from $1.31(1) \mathrm{meV}$ at $0 \mathrm{GPa}$ to $0.82(2) \mathrm{meV}$ at $1.9 \mathrm{GPa}$. However, the analysis of the temperature dependence of $\sigma_{\mathrm{sc}}(T)$ reflects only a $\sim 5 \%$ increase of the zerotemperature value of $n_{s}$. This means that the absolute value of the superfluid density in $2 \mathrm{M}-\mathrm{WS}_{2}$ is very weakly affected by pressure. The band structures, calculated in the presence of the spin-orbit coupling for ambient pressure and under the applied pressures of $p=1.3 \mathrm{GPa}$ and $1.9 \mathrm{GPa}$ are shown in Fig. $4 \mathrm{~d}-\mathrm{f}$ (see also Fig. S1 in the Supplementary Information). The band inversion between the $p$-orbital of the $\mathrm{S}$ atoms and the $d$-orbital of the $\mathrm{W}$ atoms at the $\Gamma$ point is found up to the highest investigated pressure. This indicates that the topology of the bands in $2 \mathrm{M}-\mathrm{WS}_{2}$ remains nontrivial under pressure. We also find that the electron pockets at the $\Gamma$-point shrink upon application of pressure, which might have some interesting consequences on the physical properties of the system.

\section{DISCUSSION}

We can now discuss the main results of our measurements on $2 \mathrm{M}$ $\mathrm{WS}_{2}$ :

(i) The temperature dependence of the superfluid density, reflected by the one of the relaxation rate $\sigma_{\mathrm{sc}}$ is well described at all pressures by a single gap s-wave model. This is surprising since a multi-band bulk electronic structure with hole-like bands around the $N$ point and electron-like bands around the $\Gamma$ point was found for $2 \mathrm{M}-\mathrm{WS}_{2}$. On the other hand, Hall effect measurements showed that the holetype carriers are dominant with a very high density of $n_{e, h}=$ $8.58 \times 10^{26} \mathrm{~m}^{-3}$ at $T=10 \mathrm{~K}$. It is interesting that the superfluid density $n_{s} /\left(m^{*} / m_{e}\right) \simeq 2.8 \times 10^{26} \mathrm{~m}^{-3}$, which we estimated for $2 \mathrm{M}-\mathrm{WS}_{2}$ is very close to the normal state hole density $n_{e, h}$ indicating that the superconductivity stems mostly from hole carriers. Thus, single gap superconductivity in this multi-band system may be explained by the fact that the superconducting gap occurs only on the hole-like Fermi surface and that the carrier density on the electron Fermi surface is not sufficient to induce superconductivity. This conclusion is also substantiated by the fact that superfluid density is insensitive to the shrinkage of the electron pocket, caused by pressure. If superconductivity would exist on the electron pocket, the pressure induced reduction of the size of the electron pocket would result to the reduction of the associated electron carrier concentration and thus the reduction of the superfluid density, which is not the case in $2 \mathrm{M}-\mathrm{WS}_{2}$. Single gap s-wave superconductivity in $2 \mathrm{M}-\mathrm{WS}_{2}$ is also different from the two-gap $s+s$-wave superconductivity, observed in the superconducting TMDs $T_{\mathrm{d}}-\mathrm{MoTe}_{2}$ and 

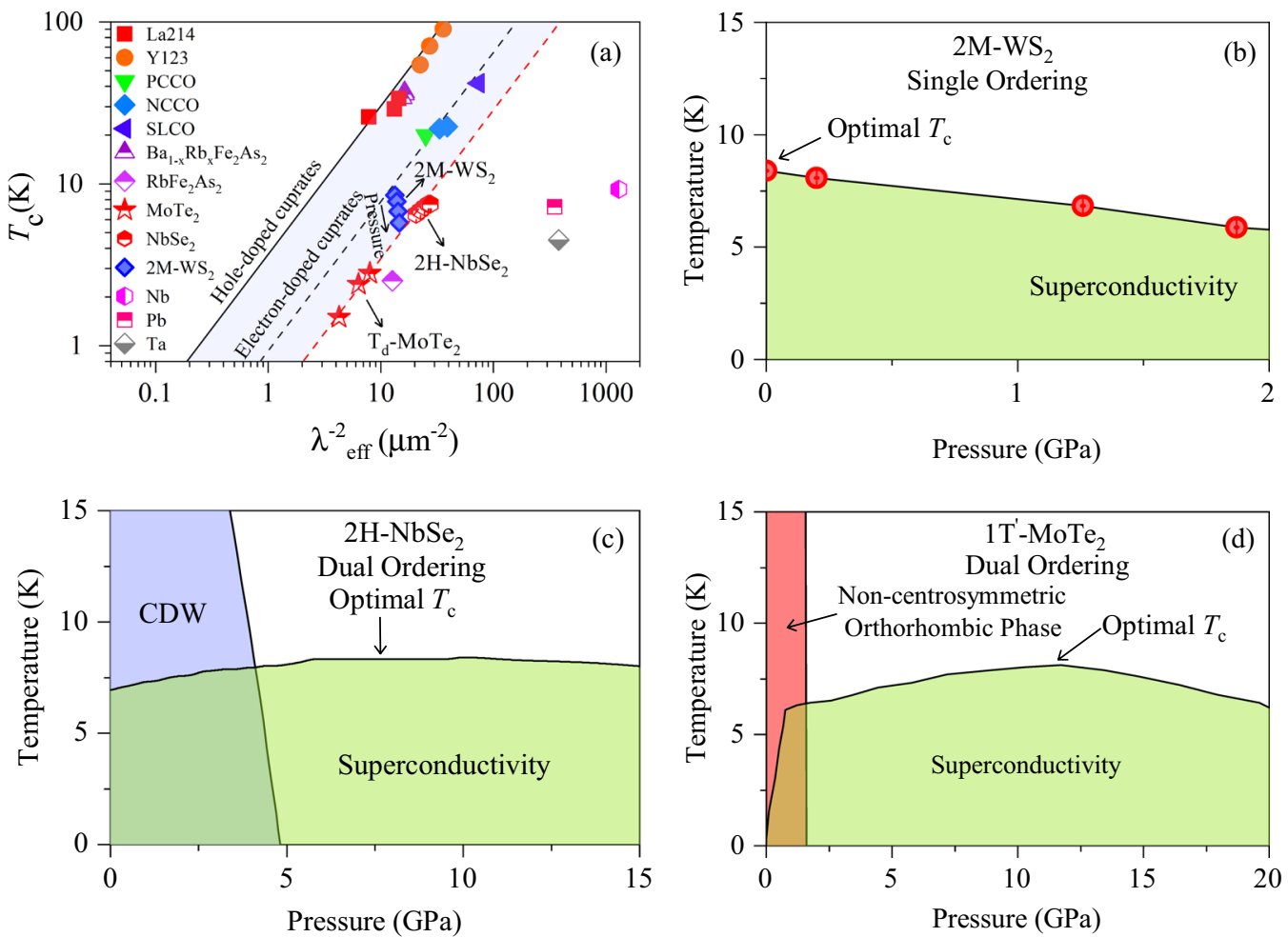

Fig. 5 Superfluid Density versus $T_{c}$ and comparison between the phase diagrams. a A plot of $T_{c}$ against the $\lambda^{-2}(0)$ obtained from our $\mu$ SR experiments in $2 \mathrm{M}-\mathrm{WS}_{2}, 2 \mathrm{H}-\mathrm{NbSe}_{2}$ and $\mathrm{MoTe}_{2}$. Data points for $\mathrm{MoTe}_{2}$ and $2 \mathrm{H}-\mathrm{NbSe}_{2}$ are taken from refs. ${ }^{23,24}$. The dashed red line represents the linear fit to the $\mathrm{MoTe}_{2}$ and $2 \mathrm{H}-\mathrm{NbSe}_{2}$ data. Uemura relation for hole and electron-doped cuprates are shown as solid ${ }^{25,26,28}$ and dashed lines, $^{30}$ respectively. Few points for cuprate and Fe-based superconductors are shown. The points for various conventional BCS superconductors are also shown. The temperature-pressure phase diagram for $2 \mathrm{M}-\mathrm{WS}_{2} \mathbf{b}$, showing only the superconducting order. The schematic temperature-pressure phase diagrams for $2 \mathrm{H}-\mathrm{NbSe}_{2}$ c (after ref. ${ }^{53}$ ) and $\mathrm{MoTe}_{2}$ (after ref. ${ }^{5}$ ) d, exhibiting dual ordering

$2 \mathrm{H}-\mathrm{NbSe}_{2}$. This again might be related to the fact that in both these systems both hole and electron bands contribute to superconductivity, while in $2 \mathrm{M}-\mathrm{WS}_{2}$ the superconductivity most likely arises predominantly from the hole like band. Regarding the surface states, a single Dirac cone at the $\Gamma$ point was reported. When the Fermi level is exactly at the Dirac point, only a single Fermi surface was obtained. ${ }^{35} \mathrm{~A}$ single Fermi surface at the surface and a dominant hole-like Fermi surface in the bulk is consistent with single gap superconductivity, observed in our study. It was also suggested that if the superconductivity is induced on this single Fermi surface, topological superconductivity is expected. As the muons penetrate deep into the material, we cannot obtain information on the superconductivity on the surface and about its topological nature. However, because of the semimetallic bulk electronic structure, the topological surface states are partially mixed with bulk states. Thus, the superconducting properties in the bulk could, to some extent, reflect the properties also on the surface.

(ii) We observed, for the first time, a strong negative pressure effect on the critical temperature $T_{c}$ and the superconducting gap $\Delta$. Both quantities decrease by $\sim 30 \%$ under $1.9 \mathrm{GPa}$. Note that the superconducting transition temperature, estimated by the Allen and Dynes modified McMillan formula, ${ }^{35}$ was found to be $\sim 2 \mathrm{~K}$, which is a factor of 4 smaller than the experimental value $T_{\mathrm{c}} \simeq 8.5 \mathrm{~K}$ (see Fig. $\mathrm{S} 2$ in the Supplementary Information). This indicates that some dynamical and anharmonic effects on the electron-phonon coupling as well as interband scattering between electron and hole pockets are most likely the reasons for high- $T_{\mathrm{c}}$ in $2 \mathrm{M}-\mathrm{WS}_{2}$. According to our DFT calculations, the electron pocket around the $\Gamma$ point shrinks by pressure, which would lead to the suppression of interband processes and this might have some effect on $T_{\mathrm{c}}$. Pressure could also weaken the anharmonic electron-lattice interactions, giving rise to the suppression of $T_{\mathrm{c}}$. Some additional experiments are certainly needed to understand the negative pressure effect on the critical temperature. The superfluid density $n_{s} /\left(\mathrm{m}^{*} /\right.$ $m_{e}$ ) is essentially pressure independent, displaying at the most a slight increase of about $5 \%$. The ratio between the superfluid density and the critical temperature is located in the Uemura plot close to those of $T_{\mathrm{d}}-\mathrm{MoTe}_{2}$ and $2 \mathrm{H}-\mathrm{NbSe}_{2}$ and other unconventional superconductors, as shown in Fig. 5 . This suggests that $2 \mathrm{M}-\mathrm{WS}_{2}$ exhibits rather unconventional SC properties and might suggest a common mechanism and related electronic origin for the superconductivity in these superconducting TMDs. On the other hand, the insensitivity of the superfluid density to the strong change in $T_{c}$ is in contrast to the strong correlation between these two quantities in $T_{\mathrm{d}}-\mathrm{MoTe}_{2}$ and $2 \mathrm{H}-\mathrm{NbSe}_{2}$. Let us try to understand this apparent discrepancy. The nearly linear relationship between $T_{\mathrm{c}}$ and the superfluid density was originally observed in hole-doped cuprates in the underdoped region of the phase diagram, ${ }^{25,26}$ where the ratio between $T_{\mathrm{C}}$ and their effective Fermi temperature $T_{\mathrm{F}}$ is about $T_{\mathrm{C}} / T_{\mathrm{F}} \sim 0.05$ corresponding to a 4-5 times reduction of $T_{\mathrm{C}}$ from the ideal Bose condensation temperature for a noninteracting Bose gas. These results were discussed in terms of a crossover from Bose-Einstein condensation to BCS-like condensation. ${ }^{28}$ It is important to note that in cuprates the relationship between $T_{c}$ and $n_{s} /\left(m^{*} / m_{e}\right)$ was shown to depend on which part of the SC dome the samples are located. Thus, the left-side of the dome (underdoped), which 
has close proximity with the competing/cooperating antiferromagnetic phase, the center of the dome (optimally doped), which is away from the AFM phase or the right side of the dome (overdoped) region, which is clearly seperated from the AFM phase. The linear increase of $T_{c}$ with $n_{s} /\left(m^{*} /\right.$ $m_{e}$ ) is observed only in the underdoped region of the phase diagram. Within the optimal doping region $T_{c}$ does not change despite the fact that $n_{s} /\left(\mathrm{m}^{*} / \mathrm{m}_{e}\right)$ increases. In the overdoped region, $T_{c}$ decreases, but $n_{s} /\left(m^{*} / m_{e}\right)$ shows a small increase only. A similar behaviour was also observed in Fe-based superconductors. ${ }^{52}$ With this in mind, we compare the temperature-pressure phase diagrams for the systems $2 \mathrm{M}-\mathrm{WS}_{2}, T_{\mathrm{d}}-\mathrm{MoTe}_{2}, 2 \mathrm{H}-\mathrm{NbSe}$. The phase diagrams are shown in Fig. $5 b-d$. There are two obvious differences between the phase diagrams. In the case of $2 \mathrm{H}-\mathrm{NbSe}_{2}$ and $T_{\mathrm{d}}-\mathrm{MoTe}_{2}$ (Fig. $5 \mathrm{C}, \mathrm{d}$ ), the phase diagram consists of two different orders at ambient pressure: superconductivity and either charge density wave order or non-centrosymmetric structural order. In addition, $T_{\mathrm{c}}$ of both systems increases with increasing pressure within the investigated pressure region $(0-2 \mathrm{GPa})$, indicating that the samples are located in the left-hand side of the dome with close proximity to another phase. In the case of $2 \mathrm{M}-\mathrm{WS}_{2}$ (Fig. 5b), the system has solely superconducting order and there is no additional competing/cooperating order. In addition, the system $2 \mathrm{M}$ $\mathrm{WS}_{2}$ has the highest $T_{\mathrm{c}}$ at ambient pressure, suggesting that the system is already in its optimal conditions in terms of superconductivity. Upon application of pressure, $T_{\mathrm{c}}$ decreases substantially, pushing the system towards the right-hand side of the dome, i.e. pushing it even further away from any possible competing phase. So, the location of $2 \mathrm{M}-\mathrm{WS}_{2}$ in the optimal (ambient pressure) and above the optimal (under pressure) superconducting regions of the phase diagram might be a reason for the absence of the correlation between the superfluid density and the critical temperature in this system.

In conclusion, using DFT, we show that the topology of the bands in $2 \mathrm{M}-\mathrm{WS}_{2}$ remains nontrivial up to the highest applied pressure. We further provide the first microscopic investigation of the superconductivity in the layered superconductor $2 \mathrm{M}-\mathrm{WS}_{2}$ at ambient pressure as well as under hydrostatic pressures by means of muon-spin rotation. Specifically, the zero-temperature magnetic penetration depth $\lambda(0)$ and the temperature dependence of the superfluid density were studied in $2 \mathrm{M}-\mathrm{WS}_{2}$ by means of $\mu \mathrm{SR}$ experiments with pressures up to $p \simeq 1.9 \mathrm{GPa}$. The temperature dependence of the superfluid density $\lambda^{-2}$ is well described by a single gap s-wave scenario with a gap value of $\Delta \simeq 1.31$ (1) meV at ambient pressure. Considering the previous observations of the dominant hole-like Fermi surface in the bulk and single Fermi surface on the surface we suggest that the well resolvable SC gap occurs only on the hole-like Fermi surface. We also find that the ratio $T_{\mathrm{c}} / n_{\mathrm{s}}$ is located in the Uemura plot close to those of $T_{\mathrm{d}}-\mathrm{MoTe}_{2}$ and $2 \mathrm{H}-\mathrm{NbSe}_{2}$ and other unconventional superconductors, suggesting that $2 \mathrm{M}-\mathrm{WS}_{2}$ exhibits non-standard SC properties. Moreover, we observed the strong negative pressure effect on $T_{\mathrm{c}}$ and $\Delta$, which may partly be due to the reduction of the size of the electron pocket at the $\Gamma$ point, at which a band inversion appears up to the highest applied pressure. The superfluid density shows only a very weak positive pressure effect and thus the correlation between the superfluid density and the critical temperature is absent in $2 \mathrm{M}-\mathrm{WS}_{2}$. This is in contrast to the strong pressure effects on $\lambda^{-2}$ and its correlation with $T_{c}$, observed in the superconducting TMDs $T_{\mathrm{d}}-\mathrm{MoTe}_{2}$ and $2 \mathrm{H}-\mathrm{NbSe}_{2}$. This discrepancy is discussed in terms of the absence of competing or cooperating order to superconductivity in $2 \mathrm{M}-\mathrm{WS}_{2}$. These results hint towards the complex nature of the superconductivity in TMDs, despite its s-wave nature, which might have far reaching consequences for the future development of devices based on these materials.

\section{METHODS}

\section{Sample preparation}

Topochemical synthesis procedure of the metastable, monoclinic tungsten diselenide $\left(2 \mathrm{M}-\mathrm{WS}_{2}\right)$ can be found elsewhere. ${ }^{35,54}$ In a first step, a hexagonal phase of $2 \mathrm{M}-\mathrm{WS}_{2}\left(\mathrm{SG} \mathrm{P}_{3} / \mathrm{mmc}\right.$ - No 194), was synthetized by solid state reaction method at $850^{\circ} \mathrm{C}$ for $35 \mathrm{~h}$ from a stoichiometric mixture of elemental sulfur ( $5 \mathrm{~N}$, Alfa Aesar) and tungsten ( $5 \mathrm{~N}$, Alfa Aesar). Than potassium intercalation and growth of $\mathrm{KWS}_{2}$ was conducted by annealing the stoichiometric mixture of $\mathrm{K}\left(3 \mathrm{~N} 5\right.$, Aldrich) and $2 \mathrm{M}-\mathrm{WS}_{2}$ at $850^{\circ} \mathrm{C}$. After $35 \mathrm{~h}$ a specimen was cooled down to $550^{\circ} \mathrm{C}$ with a rate of $6{ }^{\circ} \mathrm{C} / \mathrm{h}$. All syntheses reactions and sample growth processes described above where conducted in a double wall, evacuated and sealed quartz ampules. In a third step $\mathrm{K}_{x}\left(\mathrm{H}_{2} \mathrm{O}\right) y W \mathrm{~S}_{2}$ compound was created by washing $3 \mathrm{~g}$ of $\mathrm{KWS}_{2}$ with deionized water. Finally $\mathrm{K}_{x}\left(\mathrm{H}_{2} \mathrm{O}\right) \mathrm{yWS}_{2}$ was oxidized with $200 \mathrm{ml}$ of $0.1 \mathrm{~N} \mathrm{~K}_{2} \mathrm{Cr}_{2} \mathrm{O}_{7} / 0.1 \mathrm{~N} \mathrm{H}_{2} \mathrm{SO}_{4}$ aqueous solution in order to get polycrystalline samples of $2 \mathrm{M}_{-}-\mathrm{WS}_{2}$. Powders from the same batch were pressed in the form of pellets and were measured with various instruments. For the low background GPS instrument and low-temperature HAL-9500 instrument, equipped with BlueFors vacuum-loaded cryogen-free dilution refrigerator (DR), we used one pellet with $10 \mathrm{~mm}$ in diameter. For high pressure GPD instrument, several pellets with $5.9 \mathrm{~mm}$ in diameter were prepared and placed inside the pressure cell.

\section{Pressure cell}

Double wall piston-cylinder type of cell made of CuBe/MP35N material was used in $\mu \mathrm{SR}$ experiments to generate pressures up to $1.9 \mathrm{GPa} .^{39-42}$ Small indium plate was placed together with the sample in the pressure cell filled with the Daphne oil. The pressure was estimated by tracking the SC transition of a indium plate by AC susceptibility. The filling factor of the pressure cell was maximised. The fraction of the muons stopping in the sample was $\sim 45 \%$. The background of the pressure cell is well known and characterised. $^{39}$

\section{$\mu \mathrm{SR}$ experiment}

Spin-polarized muons $\mu^{+}$are extremely sensitive magnetic probes. Muonspin experiences the Larmor precession either in the local field or in an applied magnetic field. By probing the field distribution in the vortex state of a Type-II superconductor using transverse field $\mu \mathrm{SR}$ technique, fundamental parameters such as the magnetic penetration depth $\lambda$ and the coherence length $\xi$ can be measured in the bulk of a superconductor.

Analysis of TF- $\mu$ SR data

The following function is used to analyse the TF $\mu$ SR data: ${ }^{55,56}$

$$
\begin{aligned}
P(t)= & A_{s} \exp \left[-\frac{\left(\sigma_{s c}^{2}+\sigma_{n m}^{2}\right) t^{2}}{2}\right] \cos \left(\gamma_{\mu} B_{i n t, s} t+\varphi\right) \\
& +A_{p c} \exp \left[-\frac{\sigma_{p c}^{2} t^{2}}{2}\right] \cos \left(\gamma_{\mu} B_{i n t, p c} t+\varphi\right),
\end{aligned}
$$

Here $A_{s}$ and $A_{p c}$ are the initial asymmetries of the $\mu \mathrm{SR}$ signal for the sample and the pressure cell, respectively. $\gamma /(2 \pi) \simeq 135.5 \mathrm{MHz} / \mathrm{T}$ is the muon gyromagnetic ratio and $\varphi$ is the initial phase of the muon-spin ensemble. $B_{\text {int }}$ is the internal magnetic field at the muon site. The depolarization rate $\sigma_{\mathrm{sc}}$ is due to the formation of the flux line lattice in the SC state and $\sigma_{\mathrm{nm}}$ describes the damping due to the nuclear dipolar contribution. $\sigma_{\mathrm{nm}}$ was obtained above $T_{\mathrm{c}}$ and was assumed to be constant over the entire temperature range. The relaxation rate $\sigma_{\mathrm{pc}}$ reflects the depolarization due to the nuclear moments of the pressure cell. It exhibits the temperature dependence below $T_{c}^{41}$ due to the influence of the diamagnetic response of the SC sample on the pressure cell. In order to consider this influence we assume a linear coupling between $\sigma_{\mathrm{pc}}$ and the field shift of the internal magnetic field in the SC state: $\sigma_{\mathrm{pc}}(T)=\sigma_{\mathrm{pc}}\left(T>T_{\mathrm{c}}\right)$ $+C(T)\left(\mu_{0} H_{\text {int,Ns }}-\mu_{0} H_{\text {int, Sc }}\right)$, where $\sigma_{\mathrm{pc}}\left(T>T_{\mathrm{c}}\right)=0.25 \mu \mathrm{s}^{-1}$ is the temperature independent relaxation rate. $\mu_{0} H_{\text {int,Ns }}$ and $\mu_{0} H_{\text {int,Sc }}$ are the internal magnetic fields measured in the normal and in the SC state, respectively. 
Analysis of $\lambda(T)$

$\lambda(T)$ was calculated within the London approximation $(\lambda \gg \zeta)$ by the following function: ${ }^{50,55}$

$\frac{\lambda^{-2}\left(T, \Delta_{0, i}\right)}{\lambda^{-2}\left(0, \Delta_{0, i}\right)}=1+\frac{1}{\pi} \int_{0}^{2 \pi} \int_{\Delta(T, \varphi)}^{\infty}\left(\frac{\partial f}{\partial E}\right) \frac{E d E d \varphi}{\sqrt{E^{2}-\Delta_{i}(T, \varphi)^{2}}}$,

where $f=\left[1+\exp \left(E / k_{\mathrm{B}} T\right)\right]^{-1}$ is the Fermi function, $\varphi$ is the angle along the Fermi surface, and $\Delta_{i}(T, \varphi)=\Delta_{0, i} \Gamma\left(T / T_{c}\right) g(\varphi)\left(\Delta_{0, i}\right.$ is the maximum gap value at $T=0)$. The temperature dependence of the gap is given by the expression $\Gamma\left(T / T_{\mathrm{c}}\right)=\tanh \left\{1.82\left[1.018\left(T_{\mathrm{c}} / T-1\right)\right]^{0.51}\right\}^{57}$ while $g(\varphi)$ describes the angular dependence of the gap. $g(\varphi)=1$ for both an $s$-wave and an $s+s$-wave gap, and $g(\varphi)=|\cos (2 \varphi)|$ for a $d$-wave gap.

\section{DFT calculations}

$2 \mathrm{M}-\mathrm{WS}_{2}$ crystal has a monoclinic structure with the space group symmetry $C_{2 / m}$ (number 12) and the following lattice parameters $a=12.856 \AA$, $b=$ $3.2257 \AA, c=5.7109 \AA, a=\gamma=90^{\circ}$ and $\beta=112.942^{\circ}$ (crystallographic data CCDC 1853656). Full-relativistic electronic structure calculations were performed in Vienna $a b$ initio simulation package (VASP) ${ }^{58,59}$ using Perdew-Burke-Ernzerhof $(\mathrm{PBE})^{60,61}$ generalized gradient approximation (GGA) exchange-correlation functional and projected augmented wave (PAW) potentials. ${ }^{62,63}$ The van der Waals interactions were taken into account within the DFT-D2 approach by Grimme. ${ }^{64}$ The plane wave basis cutoff was set to $600 \mathrm{eV}$ and $9 \times 9 \times 9 \mathrm{k}$-points grids were generated within Monkhorst-Pack method.

\section{DATA AVAILABILITY}

All relevant data are available from the authors. The data can also be found at the following link http://musruser.psi.ch/cgi-bin/SearchDB.cgi using the following details: Area: HAL and GPD. Year: 2018. Run numbers: 1009-1042 for HAL and 3199-3281 for GPD.

\section{ACKNOWLEDGEMENTS}

The $\mu \mathrm{SR}$ experiments were carried out at the Swiss Muon Source $(\mathrm{S} \mu \mathrm{S})$, Paul Scherrer Insitute, Villigen, Switzerland using the HAL-9500 $\mu$ SR spectrometer ( $\pi$ E3 beamline), GPS instrument ( $\pi \mathrm{M} 3$ beamline) and high pressure GPD instrument ( $\mu \mathrm{E} 1$ beamline). The work at the University of Zürich was supported by the Swiss National Science Foundation under Grant No. PZ00P2_174015. R.K. acknowledges the Swiss National Science Foundation (grants 200021_149486 and 200021_175935). T.N. and S.S.T. acknowledge the funding from the European Research Council (ERC) under the European Union's Horizon 2020 research and innovation programm (ERC-StGNeupert-757867-PARATOP). M.Z.H. is supported by US DOE/BES grant no. DE-FG-0205ER46200.

\section{AUTHOR CONTRIBUTIONS}

Project planning and coordination: Z.G. Sample growth and XRD experiment: D.G. and E.P. $\mu S R$ experiments: Z.G., R.K., J.V., H.L., A.A. $\mu$ SR data analysis: Z.G. Magnetization measurements: F.v.R. DFT calculations: M.B., S.S.T. and T.N. Data interpretation and draft writing: Z.G. with contributions and comments from all authors.

\section{ADDITIONAL INFORMATION}

Supplementary information accompanies the paper on the npj Quantum Materials website (https://doi.org/10.1038/s41535-019-0189-5).

Competing interests: The authors declare no competing interests.

Publisher's note: Springer Nature remains neutral with regard to jurisdictional claims in published maps and institutional affiliations.

\section{REFERENCES}

1. Soluyanov, A. et al. Type II Weyl Semimetals. Nature 527, 495-498 (2015).

2. Huang, L. et al. Spectroscopic evidence for a type II Weyl semimetallic state in $\mathrm{MoTe}_{2}$. Nat. Mater. 15, 1155-1160 (2016).

3. Xu, X., Yao, W., Xiao, D. \& Heinz, T. F. Spin and pseudospins in layered transition metal dichalcogenides. Nat. Phys. 10, 343-350 (2014).
4. Ali, M. N. et al. Large, non-saturating magnetoresistance in $\mathrm{WTe}_{2}$. Nature 514, 205-208 (2014).

5. Qi, Y. et al. Superconductivity in Weyl semimetal candidate $\mathrm{MoTe}_{2}$. Nat. Commun 7, 11038 (2016).

6. Qian, X., Liu, J., Fu, L. \& Li, J. Quantum spin Hall effect in two-dimensional transition metal dichalcogenides. Science 346, 1344-1347 (2014).

7. Zhang, Y. J., Oka, T., Suzuki, R., Ye, J. T. \& Iwasa, Y. Electrically switchable chiral light-emitting transistor. Science 344, 725-728 (2014).

8. Ugeda, M. M. et al. Characterization of collective ground states in single-layer $\mathrm{NbSe}_{2}$. Nat. Phys. 12, 92-97 (2016).

9. Runze, Y. et al. Absence of local fluctuating dimers in superconducting $\operatorname{Ir}_{1-x}(\mathrm{Pt}$, $\mathrm{Rh})_{x} \mathrm{Te}_{2}$. Phys. Rev. B 97, 174515 (2018).

10. Sun, Y., Wu, S. C., Ali, M. N., Felser, C. \& Yan, B. Prediction of Weyl semimetal in orthorhombic MoTe 2 . Phys. Rev. B 92, 161107 (2015).

11. Belopolski, I. et al. Discovery of a new type of topological Weyl fermion semimetal state in $\mathrm{Mo}_{x} \mathrm{~W}_{1-x} \mathrm{Te}_{2}$. Nat. Commun. 7, 13643 (2016).

12. Le, L. P. et al. Magnetic penetration depth in layered compound $\mathrm{NbSe}_{2}$ measured by muon spin relaxation. Physica C 185, 2715-2716 (1991).

13. Yue, Y. \& Kivelson, S. A. Fragile superconductivity in the presence of weakly disordered charge density waves. Phys. Rev. B 99, 144513 (2019).

14. Yokoya, T. et al. Fermi surface sheet-dependent superconductivity in $2 \mathrm{H}-\mathrm{NbSe}_{2}$. Science 294, 2518-2520 (2001).

15. Sipos, B. et al. From Mott state to superconductivity in $1 \mathrm{~T}-\mathrm{TaS}_{2}$. Nat. Mat. $\mathbf{7}$, 960-965 (2008).

16. Tidman, J. P., Singh, O., Curzon, A. E. \& Frindt, R. F. The phase transition in $2 \mathrm{H}-\mathrm{TaS}_{2}$ at 75K. Philos. Mag. 30, 1191-1194 (1974).

17. Thompson, A. H., Gamble, F. R. \& Koehler, R. H. Jr. Effects of intercalation on electron transport in tantalum disulfide. Phys. Rev. B 5, 2811-2816 (1972).

18. Naito, M. \& Tanaka, S. Electrical transport properties in $2 \mathrm{H}-\mathrm{NbS}_{2},-\mathrm{NbSe}_{2},-\mathrm{TaS}_{2}$ and -TaSe 2 . J. Phys. Soc. Jpn 51, 219-227 (1982).

19. Ye, J. T. et al. Superconducting dome in a gate-tuned band insulator. Science $\mathbf{3 3 8}$, 1193-1196 (2012).

20. Shi, W. et al. Superconductivity series in transition metal dichalcogenides by ionic gating. Sci. Rep. 5, 12534 (2015).

21. Klemm, R. A. Pristine and intercalated transition metal dichalcogenide superconductors. Physica C 514, 86-94 (2015).

22. Lu, J. et al. Full superconducting dome of strong Ising protection in gated monolayer WS 2 . Proc. Natl Acad. Sci. USA 115, 3551-3556 (2018).

23. Guguchia, Z. et al. Signatures of the topological $s^{+-}$superconducting order parameter in the type-II Weyl semimetal $T_{d}-\mathrm{MoTe}_{2}$. Nat. Commun. 8, 1082 (2017).

24. Rohr, F. V. et al. Unconventional scaling of the superfluid density with the critical temperature in transition metal dichalcogenides. Preprint at https://arxiv.org/abs/ 1903.05292.

25. Uemura, Y. J. et al. Universal correlations between $T_{\mathrm{c}}$ and $n_{\mathrm{s}} / m^{*}$ (carrier density over effective mass) in High- $T_{c}$ cuprate superconductors. Phys. Rev. Lett. 62, 2317-2320 (1989).

26. Uemura, $\mathrm{Y}$. J. et al. Magnetic-field penetration depth in $\mathrm{K}_{3} \mathrm{C}_{60}$ measured by muon spin relaxation. Nature 352, 605-607 (1991).

27. Uemura, Y. J. Superconductivity: exotic commonalities in phase and mode. Nat. Mater. 8, 253-255 (2009).

28. Uemura, Y. J. et al. Basic similarities among cuprate, bismuthate, organic, chevre phase, and heavy-fermion superconductors shown by penetration depth measurements. Phys. Rev. Lett. 66, 2665-2668 (1991).

29. Emery, V. \& Kivelson, S. Importance of phase fluctuations in superconductors with small superfluid density. Nature 374, 434-437 (1995).

30. Shengelaya, A. et al. Muon-spin-rotation measurements of the penetration depth of the infinite-layer electron-doped $\mathrm{Sr}_{0.9} \mathrm{La}_{0.1} \mathrm{CuO}_{2}$ cuprate superconductor. Phys. Rev. Lett. 94, 127001 (2005).

31. Luetkens, $\mathrm{H}$. et al. The electronic phase diagram of the $\mathrm{LaO}_{1{ }_{1} x} \mathrm{~F}_{x} \mathrm{FeAs}$ superconductor. Nat. Mater. 8, 305-309 (2009).

32. Carlo, J. P. et al. Static magnetic order and superfluid density of $R F e A s(O, F)(R=L a$, $\mathrm{Nd}, \mathrm{Ce}$ ) and LaFePO studied by muon spin relaxation: unusual similarities with the behavior of cuprate superconductors. Phys. Rev. Lett. 102, 087001 (2009).

33. Khasanov, R. et al. Muon spin rotation studies of $\mathrm{SmFeAsO}_{0.85}$ and $\mathrm{NdFeAsO}_{0.85}$ superconductors. Phys. Rev. B 78, 092506 (2008).

34. Guguchia, Z. et al. Muon-spin rotation measurements of the magnetic penetration depth in the Fe-based superconductor $\mathrm{Ba}_{1-x} \mathrm{Rb}_{x} \mathrm{Fe}_{2} \mathrm{As}_{2}$. Phys. Rev. B 84, 094513 (2011).

35. Fang, Y. et al. Discovery of superconductivity in $2 \mathrm{M} \mathrm{WS}_{2}$ with possible topological surface states. Adv. Mater. 31, 1901942 (2019).

36. Hosur, P., Dai, X., Fang, Z. \& Qi, X.-L. Time-reversal-invariant topological superconductivity in doped Weyl semimetals. Phys. Rev. B 90, 045130 (2014).

37. Ando, Y. \& Fu, L. Topological crystalline insulators and topological superconductors: From concepts to materials. Annu. Rev. Condens. Matter Phys. 6, 361-381 (2015). 
38. Grushin, A. G. Consequences of a condensed matter realization of Lorentzviolating QED in Weyl semi-metals. Phys. Rev. D 86, 045001 (2012).

39. Khasanov, R. et al. High pressure research using muons at the Paul Scherrer Institute. High. Press. Res. 36, 140-166 (2016).

40. Andreica, D. 2001 Ph.D. thesis IPP/ETH-Zürich.

41. Maisuradze, A., Shengelaya, A., Amato, A., Pomjakushina, E. \& Keller, H. Muon spin rotation investigation of the pressure effect on the magnetic penetration depth in $\mathrm{YBa}_{2} \mathrm{Cu}_{3} \mathrm{O}_{x}$. Phys. Rev. B 84, 184523 (2011).

42. Guguchia, $Z$. et al. Direct evidence for the emergence of a pressure induced nodal superconducting gap in the iron-based superconductor $\mathrm{Ba}_{0.65} \mathrm{Rb}_{0.35} \mathrm{Fe}_{2} \mathrm{As}_{2}$. Nat. Commun. 6, 8863 (2015).

43. Sonier, J. E., Brewer, J. H. \& Kiefl, R. F. $\mu$ SR studies of the vortex state in type-II superconductors. Rev. Mod. Phys. 72, 769-811 (2000).

44. Kubo, R. \& Toyabe, T. Magnetic resonance and relaxation (North Holland, Amsterdam, 1967)

45. Guguchia, Z. et al. Probing the pairing symmetry in the over-doped Fe-based superconductor $\mathrm{Ba}_{0.35} \mathrm{Rb}_{0.65} \mathrm{Fe}_{2} \mathrm{As}_{2}$ as a function of hydrostatic pressure. Phys. Rev. B 93, 094513 (2016).

46. Khasanov, R. et al. Superconductivity and field-induced magnetism in $\mathrm{SrFe}_{1.75^{-}}$ $\mathrm{Co}_{0.25} \mathrm{As}_{2}$. Phys. Rev. Lett. 103, 067010 (2009).

47. Williams, T. J. et al. Superfluid density and field-induced magnetism in BaFe ${ }_{1}$ $\left.{ }_{-x} \mathrm{Co}_{x}\right)_{2} \mathrm{As}_{2}$ and $\left.\mathrm{SrFe}_{1-x} \mathrm{Co}_{x}\right)_{2} \mathrm{As}_{2}$ measured with muon spin relaxation. Phys. Rev. $B$ 82, 094512 (2010).

48. Sonier, J. E. et al. Magnetism and disorder effects on muon spin rotation measurements of the magnetic penetration depth in iron-arsenic superconductors. Phys. Rev. Lett. 106, 127002 (2011).

49. Brandt, E. H. Flux distribution and penetration depth measured by muon spin rotation in high- $T_{c}$ superconductors. Phys. Rev. B 37, 2349-2352 (1988).

50. Tinkham, M. Introduction to superconductivity. (Krieger Publishing Company, Malabar, Florida, 1975).

51. Frandsen, B. A. et al. Superconducting properties of noncentrosymmetric superconductor $\mathrm{CalrSi}_{3}$ investigated by muon spin relaxation and rotation. Phys. Rev. B 91, 014511 (2015).

52. Hashimoto, K. et al. A Sharp peak of the zero-temperature penetration depth at optimal composition in $\mathrm{BaFe}_{2}\left(\mathrm{As}_{1-x} \mathrm{P}_{x}\right)_{2}$. Science 336, 1554-1557 (2012).

53. Feng, Y. et al. Order parameter fluctuations at a buried quantum critical point. Proc. Natl Acad. Sci. USA 109, 7224-7229 (2012).

54. Wypych, F., Sollmann, K. \& Schollhorn, R. Metastable layered chalcogenides 1T$\mathrm{MoS}_{2}, 2 \mathrm{M}-\mathrm{WS}_{2}$ and $1 \mathrm{~T}-\mathrm{Mo}_{12} \mathrm{~W}_{12} \mathrm{~S}_{2}$ : electrochemical study on their intercalation reactions. Mater. Res. Bull. 27, 545-553 (1992).
55. Suter, A. \& Wojek, B. M. The fitting of the T-dependence of the penetration depth with a model was performed using the additional library BMW developped by B.M. Wojek. Phys. Procedia 30, 69-73 (2012).

56. Suter, A. \& Wojek, B. M. Musrfit: a free platform-independent framework for $\mu$ SR data analysis. Phys. Procedia 30, 69-73 (2012).

57. Carrington, A. \& Manzano, F. Magnetic penetration depth of $\mathrm{MgB}_{2}$. Physica C 385, 205 (2003).

58. Kresse, G. \& Furthmüller, J. Efficiency of ab-initio total energy calculations for metals and semiconductors using a plane-wave basis set. Comput. Mater. Sci. 6, 15-50 (1996).

59. Kresse, G. \& Furthmüller, J. Efficient iterative schemes for ab initio total-energy calculations using a plane-wave basis set. Phys. Rev. B 54, 11169-11186 (1996).

60. Perdew, J. P., Burke, K. \& Ernzerhof, M. Generalized gradient approximation made simple. Phys. Rev. Lett. 77, 3865-3868 (1997).

61. Perdew, J. P., Burke, K. \& Ernzerhof, M. Generalized gradient approximation made simple. Phys. Rev. Lett. 78, 1396 (1997).

62. Blöchl, P. E. Projector augmented-wave method. Phys. Rev. B 50, 17953-17979 (1994).

63. Kresse, G. \& Joubert, D. From ultrasoft pseudopotentials to the projector augmented-wave method. Phys. Rev. B 59, 1758-1775 (1999).

64. Grimme, S. Semiempirical GGA-type density functional constructed with a longrange dispersion correction. J. Comput. Chem. 27, 1787-1799 (2006).

(c) Open Access This article is licensed under a Creative Commons By Attribution 4.0 International License, which permits use, sharing, adaptation, distribution and reproduction in any medium or format, as long as you give appropriate credit to the original author(s) and the source, provide a link to the Creative Commons license, and indicate if changes were made. The images or other third party material in this article are included in the article's Creative Commons license, unless indicated otherwise in a credit line to the material. If material is not included in the article's Creative Commons license and your intended use is not permitted by statutory regulation or exceeds the permitted use, you will need to obtain permission directly from the copyright holder. To view a copy of this license, visit http://creativecommons. org/licenses/by/4.0/.

(c) The Author(s) 2019 